\title{
Design and Experimental Study of an L Shape Piezoelectric Energy Harvester
}

\author{
In-Ho Kim, ${ }^{1}$ Seon-Jun Jang, ${ }^{2}$ and Hyung-Jo Jung ${ }^{1}$ \\ ${ }^{1}$ Department of Civil and Environmental Engineering, Korea Advanced Institute of Science and Technology, \\ Daejeon 34141, Republic of Korea \\ ${ }^{2}$ School of Mechanical Engineering, Hoseo University, Asan 31499, Republic of Korea
}

Correspondence should be addressed to Seon-Jun Jang; mweagle@hoseo.edu and Hyung-Jo Jung; hjung@kaist.ac.kr

Received 24 April 2017; Revised 26 July 2017; Accepted 12 September 2017; Published 18 October 2017

Academic Editor: Toshiaki Natsuki

Copyright (C) 2017 In-Ho Kim et al. This is an open access article distributed under the Creative Commons Attribution License, which permits unrestricted use, distribution, and reproduction in any medium, provided the original work is properly cited.

\begin{abstract}
Piezoelectric energy harvesters of cantilevered beam type are studied in various fields due to simplicity. In general, these systems obtain electrical energy from mechanical strain by bending of cantilevered beam. However, conventional systems have disadvantages that they have low efficiency in frequency regions other than resonance frequency. To overcome the limitations, various energy harvesters to apply performance enhancement strategies are proposed and investigated. In this paper, a frequencychangeable L shape energy harvester which is form connected cantilever beam and rigid arm is proposed and investigated. The conventional piezoelectric energy harvester exhibits the principal frequency in the simple bending mode whereas the proposed system features the twisting mode resulting in a higher output voltage than the conventional system. The proposed energy harvester is simplified to a two-degree-of-freedom model and its dynamics are described. How the length of a rigid bar affects its natural frequencies is also studied. To evaluate the performance of the system, experiments by using a vertical shaker and numerical simulation are carried out. As a result, it is shown that the natural frequency for a twisting mode decreases as the arm length increased, and the higher output voltage is generated comparing with those of the conventional energy harvester.
\end{abstract}

\section{Introduction}

Energy harvesting technology has received strong interest in the field of structural health monitoring (SHM) based on wireless sensor networks (WSNs). Most WSNs do not guarantee long lifetime with the conventional battery technology. Furthermore, the battery requires to be frequently replaced or recharged, and connecting a sensor is difficult due to wiring. There are many potentials to apply an energy harvesting system for WSNs. The commercialized sensor system is mainly mounted on or embedded inside objects such as infrastructures and body. To ensure the implanted sensors work properly, the energy harvesting device is alternative solution for battery replacement. Among the energy sources, the vibrational energy harvester can convert ambient vibration into electrical energy. These systems are mainly operated by exploiting energy conversion mechanisms such as piezoelectric [1-3], electromagnetic [4-6], and triboelectric [7-10] effect. In comparison with the energy storage density, the electromagnetic mechanism is the highest value in the aggressive value that is theoretically possible, but the piezoelectric energy harvesting is the best in practical maximum case that is currently achievable value. In order to apply the power source for sensor, the energy harvesters of various scales are proposed and investigated. In general, the energy harvesting devices can generate hundreds of milli-Watt $(\mathrm{mW})$. The output power generated from energy harvesting system is sufficient to utilize the power supply for WSNs because the power consumption of a micro sensor is used less than $100 \mathrm{~mW}$.

In this study, the piezoelectric based energy harvester is proposed due to their high energy density [11, 12]. A variety of piezoelectric based vibrational energy harvesters have been proposed and investigated by many researchers for use of power supply in wireless applications. Typically, the vibration-induced piezoelectric energy harvester is composed of a cantilever beam with a tip mass and one or two layers of piezoelectric material (simply called unimorph or 
bimorph, resp.). Conventional energy harvester has been widely used due to its simplicity. However, these systems have many difficulties to apply in practice because power efficiency is sharply decreased except in the resonance frequency.

To overcome these limitations, many researchers proposed various energy harvesters based on improved techniques such as multimodal system, self-tuning, frequency pumping, wide-bandwidth transducers, and mechanical tuning [13-18]. The most advanced techniques are proposed to effectively generate the output power at the various frequencies of structures. Therefore, the most commonly used method in the frequency tuning is the stiffness or mass control of system. However, these systems exhibit the enhanced performance but somewhat complicated and difficult to realize. To change the stiffness or the mass of the system causes problems which are increasing static deflection and overall volume. Therefore, the frequency tuning methods through the slight structural modification are proposed and investigated.

The improved piezoelectric energy harvester which utilizes the torsional moment has been proposed. Li et al. [19] investigated a bioinspired piezo-leaf energy harvester which makes use of both bending and twisting deformations from the cross-flow. The proposed energy harvester is fabricated and experimentally investigated. In the experimental results, the piezo-leaf energy harvester has a higher power density than parallel-flow stalk-leaf system. Kim et al. [14] developed a 2DOF energy harvester which is composed of different two cantilevers in size and suspended proof mass with bearings. The 2DOF energy harvester has wider bandwidth at specific voltage level than a conventional SDOF system since translational and rotational mode are designed to be very close to each other. The torsional moment is occurred by rotational mode. Abdelkefi et al. [20, 21] conducted the numerical study for the $\mathrm{T}$ shape piezoelectric energy harvester which undergoes coupled bending and torsion by unbalanced proof masses. Similarly, a self-excited fluidic energy harvester is proposed and investigated [22, 23]. It consists of a cylinder installed to the end of piezoelectric cantilevered beam. Upstream cylinder in flow direction makes vortex behavior at the downstream. Therefore, energy harvester has coupled bending and torsional motion affected by longer cylinder than width of cantilevered beam.

In this paper, the characteristics of an L shape energy harvester are investigated and the geometrical tuning design method is proposed. The proposed energy harvester consists of a piezoelectric cantilevered beam, a rigid bar orthogonally attached to the free end of beam, and a proof mass on the rigid bar. Therefore, the system has bending motion as well as twisting motion. Moreover, the natural frequency of system can be tuned via changing the location of mass. An analytical model based on the impedance method is utilized, which is simple and easily finds the inherent physical properties and natural frequencies. In experimental study, output voltage normalized by acceleration is measured and compared. Both numerical and experimental verification were performed to show the validity of the model and power performance of the proposed device.

\section{Model of an L Shape Energy Harvester}

Proposed L shape energy harvester is composed of a cantilevered beam with a piezoelectric sheet, orthogonally attached to the rigid bar at free end of beam and a proof mass. In order to adjust mass location, foraminate rigid bar is considered for frequency tuning. The bar which is connected with a cantilever beam is considered as a rigid bar because its deformation is much smaller than that of a cantilever beam. An L shape energy harvester simultaneously plays a role of vertical and torsional springs. The schematic and simplified model of the system are illustrated in Figure 1. To portray its behavior within the extent of the linear vibration theory, a two-degree-of-freedom (i.e., 2DOF) system with an eccentric mass has been introduced. It is simplified to undamped since the natural frequencies we are concerned about are almost determined by mass and stiffness values only. It consists of two identical springs with interval $d_{1}+d_{2}$ as shown in Figure 1(b). The distance from the centerline of the cantilever to the mass is given by $(1 / 2)\left|d_{1}-d_{2}\right|$ and the moment of inertia, $J$, of the point mass is $(1 / 4) m\left(d_{1}-d_{2}\right)^{2}$.

The stiffness values are identical to a half of the bending stiffness of the cantilever and the interval $d_{1}+d_{2}$ is modulated to comply with the torsional stiffness of the cantilever. The total force $F$ applied to the system is the sum of the forces $F_{1}$ and $F_{2}$ applied to the two springs, respectively, as

$$
F=F_{1}+F_{2} \text {. }
$$

The displacement at the point mass is given by

$$
X=\frac{d_{2} X_{1}+d_{1} X_{2}}{d_{1}+d_{2}},
$$

where the displacements at points 1 and 2 are $X_{1}$ and $X_{2}$, respectively, and can be written by

$$
\begin{aligned}
& X_{1}=\frac{F_{1}}{k}, \\
& X_{2}=\frac{F_{2}}{k} .
\end{aligned}
$$

The equilibrium of moments at the point mass can be expressed as

$$
J \ddot{\theta}=F_{1} d_{1}-F_{2} d_{2} .
$$

Replacing the rotational acceleration $\ddot{\theta}$ in (4) for small angles with $\left(\ddot{X}_{2}-\ddot{X}_{1}\right) /\left(d_{1}+d_{2}\right)$ and applying (3) give

$$
F_{1}\left[d_{1}-\frac{J \omega^{2}}{k\left(d_{1}+d_{2}\right)}\right]=F_{2}\left[d_{2}-\frac{J \omega^{2}}{k\left(d_{1}+d_{2}\right)}\right],
$$

where the harmonic motion is assumed as $X_{i}(t)=X_{i} \mathrm{e}^{j \omega t}$ and $\omega$ denotes the excitation frequency. The forces $F_{1}$ and $F_{2}$ can be expressed, respectively, from (1) and (5) by

$$
\begin{aligned}
& F_{1}=F \cdot \frac{k d_{2}\left(d_{1}+d_{2}\right)-J \omega^{2}}{k\left(d_{1}+d_{2}\right)^{2}-2 J \omega^{2}}, \\
& F_{2}=F \cdot \frac{k d_{1}\left(d_{1}+d_{2}\right)-J \omega^{2}}{k\left(d_{1}+d_{2}\right)^{2}-2 J \omega^{2}} .
\end{aligned}
$$




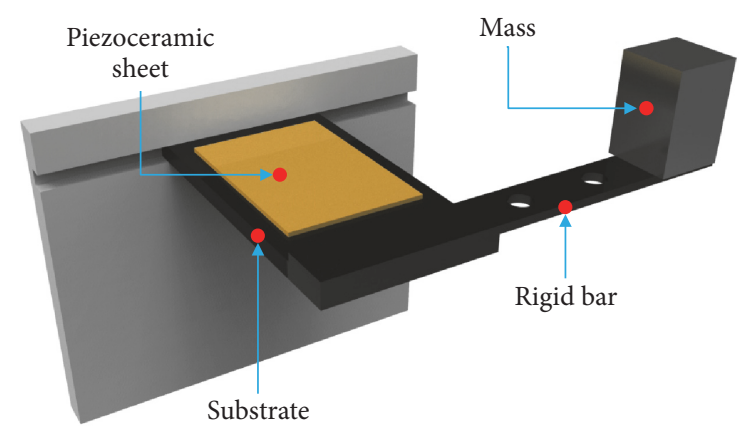

(a)

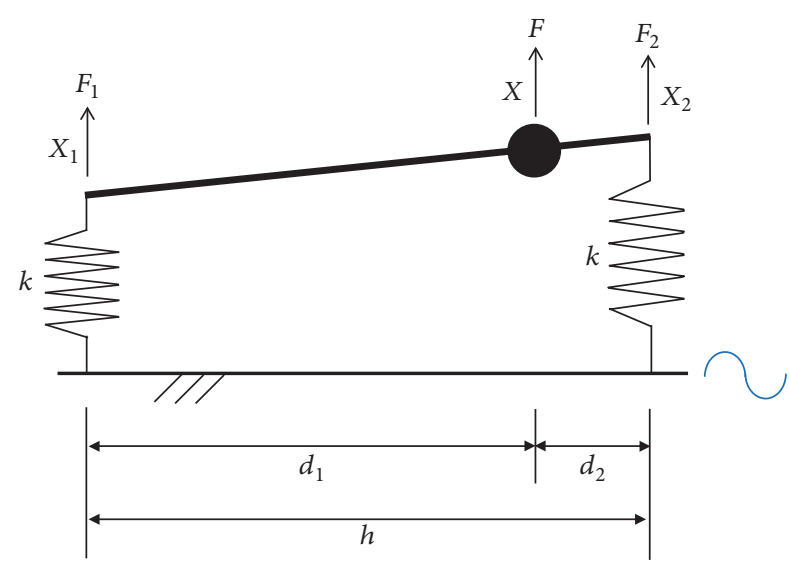

(b)

Figure 1: The proposed L shape energy harvester: (a) schematic of the proposed system and (b) simplified mechanical model.

Substituting $J=(1 / 4) m\left(d_{1}-d_{2}\right)^{2}$ and (3) and (6) into (2) can yield the displacement at the point mass in terms of constituent parameters. Then, the equivalent stiffness at the point mass becomes

$$
k_{\mathrm{eq}}=\frac{F}{X}=2 k \cdot \frac{2 k\left(d_{1}+d_{2}\right)^{2}-\left(d_{1}-d_{2}\right)^{2} m \omega^{2}}{4 k\left(d_{1}^{2}+d_{2}^{2}\right)-\left(d_{1}-d_{2}\right)^{2} m \omega^{2}} .
$$

The stiffness of (7) is the frequency dependent. Figure 2(a) shows the variation of stiffness for the three different hole locations of proof mass. In the low frequencies, the position of the proof mass affects the stiffness of the harvester. It implies that the proposed system behaves like a rotational vibrating system. In the high frequencies, the stiffness does not matter with the hole position. It works as a conventional translational system. Thus, the first and lowest mode can be found as rotational, while the second mode can be found as translational. The receptance at the point mass can be obtained from the parallel connection of a mass and the stiffness of (7) as

$$
\frac{X}{F}=\frac{1}{k_{\mathrm{eq}}-\omega^{2} m}
$$

Expanding (8) gives

$$
\frac{X}{F}=\frac{4 k\left(d_{1}^{2}+d_{2}^{2}\right)-\left(d_{1}-d_{2}\right)^{2} m \omega^{2}}{4 k^{2}\left(d_{1}+d_{2}\right)^{2}-2 k m \omega^{2}\left(3 d_{1}^{2}+3 d_{2}^{2}-2 d_{1} d_{2}\right)+m^{2} \omega^{4}\left(d_{1}-d_{2}\right)^{2}} .
$$

The two undamped natural frequencies can be found from solving the denominator of (2). The variations of the first (solid red line) and second (dashed blue line) natural frequencies for changing the distance of mass are illustrated in Figure 2(b). The vertical lines denote the locations of three holes in the rigid bar in the following experiment. The first natural frequency, which is lowest and associated with the rotational mode, is consistently decreasing with the distance $(1 / 2)\left|d_{1}-d_{2}\right|$ while the second converges. In the proposed energy harvester, thus, the geometrical tuning strategy can be established by changing the location of the proof mass on the rigid bar.

Since the first mode is rotational, the embedded piezoelectric sheets are stretched in the transverse direction of the cantilever additionally. Thus, the averaged strain value of the substrate beam is increased further, resulting in the output voltage increase.

\section{Experimental Validations of an L Shape Energy Harvester}

In order to validate the performance of an L shape energy harvester, the prototype is manufactured and installed on the vertical shaker as shown in Figure 3(a). It consists of a substrate layer (stainless steel), a proof mass (iron, $0.102 \mathrm{~kg}$ ), and the rigid bar (iron). The foraminate rigid bar is orthogonally attached at free end of beam with a proof mass. The geometric and material parameters of the system are given in Table 1 . The lead zirconate titanates (PZT-5A4H, Piezo System Co.) are used as piezoceramic sheets that are bonded to the substrate layer. Holes are located on the rigid bar to validate how the length of the rigid body affects the energy harvesting performance. The total length of the rigid bar is $140 \mathrm{~mm}$, and the gap of each hole is $30 \mathrm{~mm}$.

Figure 3(b) represents the experimental setup for performance evaluation of the proposed energy harvester. As 


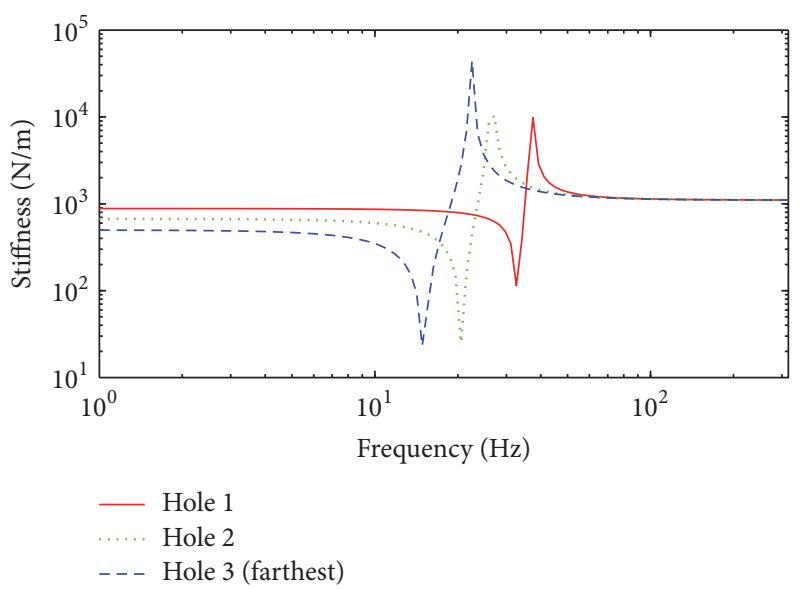

(a)

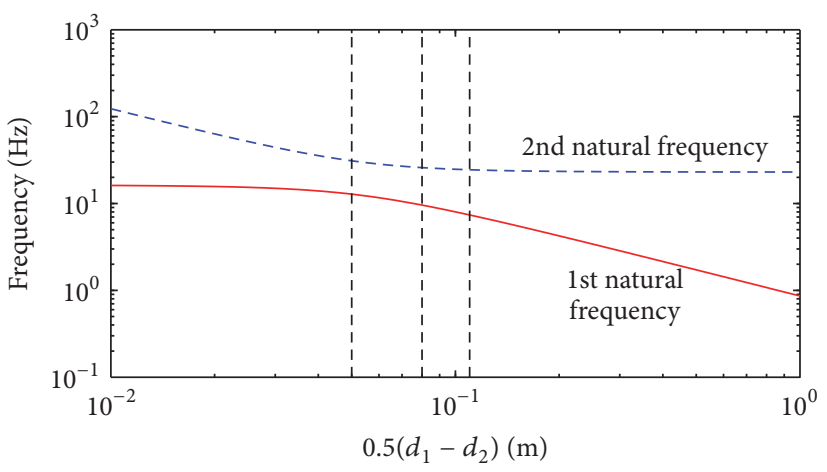

(b)

Figure 2: (a) The variation of stiffness for the three cases and (b) two undamped natural frequencies.

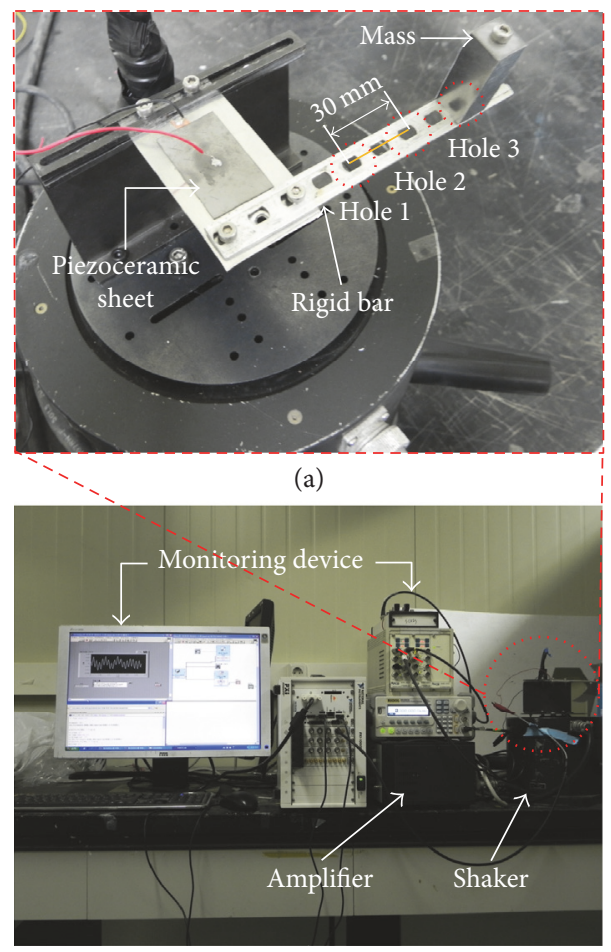

(b)

FIGURE 3: Photographs of (a) fabricated energy harvester and (b) experimental setup.

shown in the figure, the setup is composed of a function generator (AFG 3021B; Tektronix), a power amplifier (Type 2712; Brüel \& Kjær), an electrodynamic vertical shaker (Type 4808; Brüel \& Kjær), and a voltage measurement device (National Instruments). In the test, the harmonic excitation is provided by a vertical shaker. The acceleration input of the shaker is measured from an accelerometer (Model 393A03; PCB Piezotronics). The excitation frequency is varied from 1 to $20 \mathrm{~Hz}$. The frequency step of $1 \mathrm{~Hz}$ is considered in the overall range, and the step-up of $0.1 \mathrm{~Hz}$ is carried out in the vicinity of the resonance frequency. The induced openvoltage response from the piezoelectric energy harvester is measured by an oscilloscope.

Figure 4 shows the normalized output voltage in opencircuit of four different systems (i.e., the conventional system and the three proposed systems (Hole 1, Hole 2, and Hole $3)$ ). In the figure, it is observed that the proposed systems have much larger peak values than the conventional system. Particularly, the case of Hole 3 (i.e., the case that the mass is placed at the farthest location on a rigid bar) shows the largest 


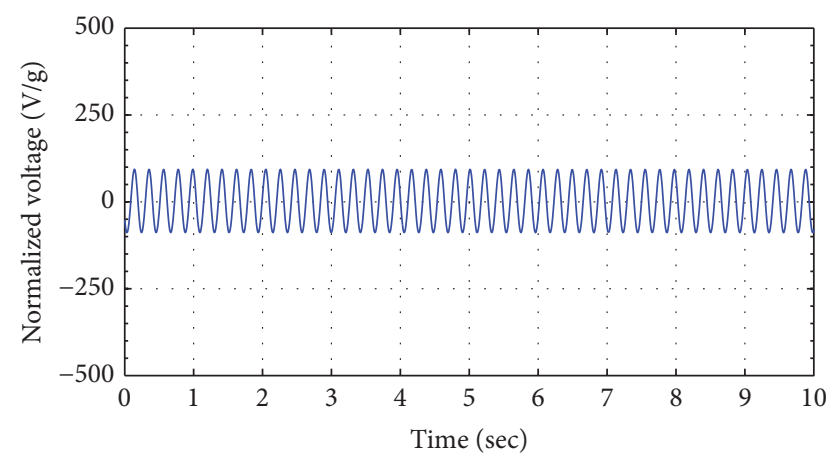

_ Conventional system

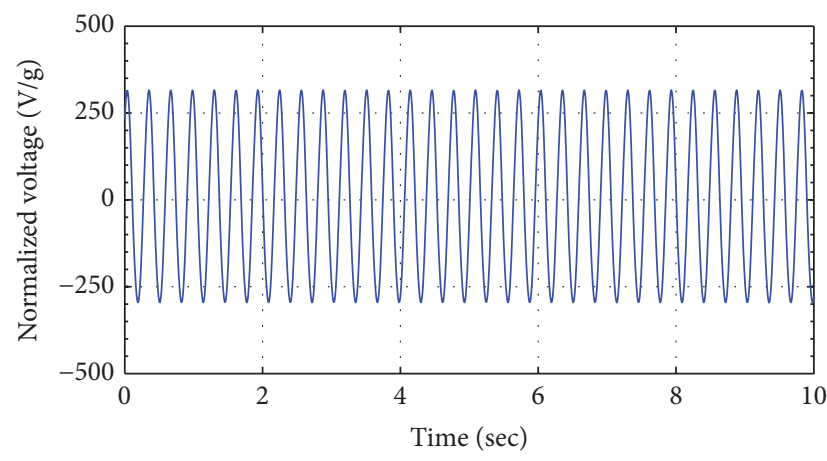

- Hole 2

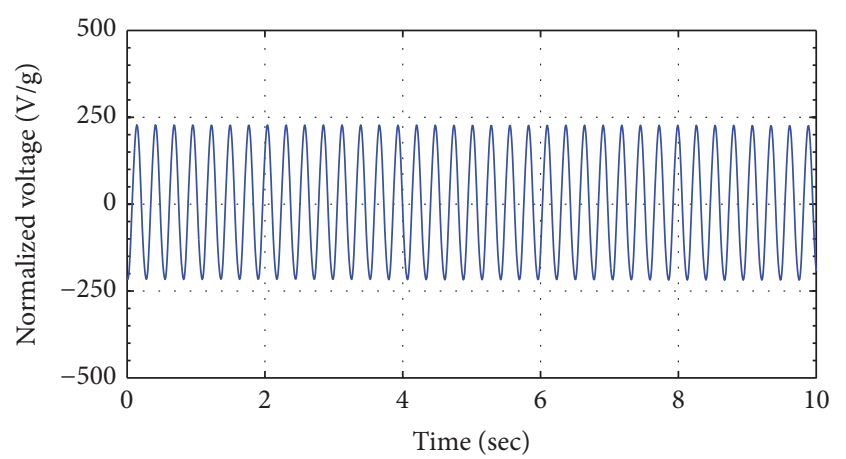

— Hole 1

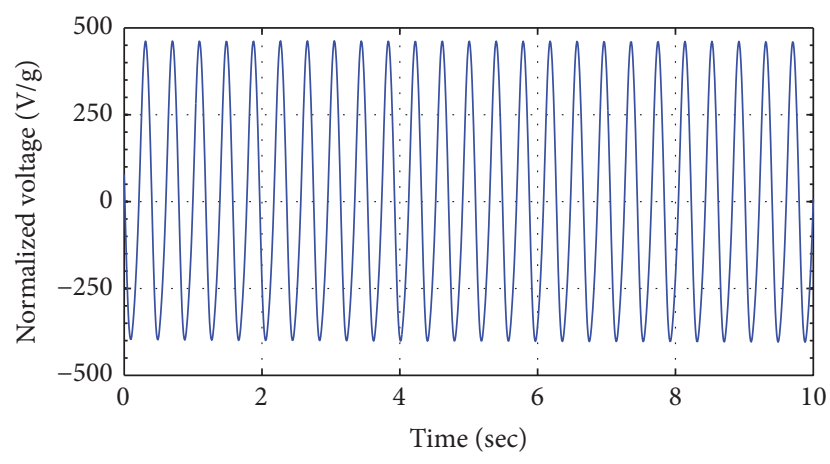

— Hole 3

FIgURE 4: Time history responses for the resonant frequencies of each case.

TABLE 1: Geometric and material parameters of the system.

\begin{tabular}{lcc}
\hline Geometric parameters & Piezoceramic & Substrate \\
\hline Size $(\mathrm{mm})$ & $25 \times 40$ & $55 \times 40$ \\
Thickness $(\mathrm{mm})$ & 0.127 & 0.2 \\
\hline Material parameters & & \\
\hline Mass density $\left(\mathrm{kg} / \mathrm{m}^{3}\right)$ & 7750 & 7850 \\
Young's modulus $(\mathrm{GPa})$ & 66 & 200 \\
$d_{31}(\mathrm{pm} / \mathrm{V})$ strain coefficient & -190 & \\
$g_{31}(\mathrm{~mm} / \mathrm{V})$ voltage coefficient & -11.6 & \\
\hline
\end{tabular}

output voltage. It is more than 4.5 times the conventional case, resulting in the enhanced performance of the proposed system. The frequency shift is also observed in the figure. The experiments show that the fundamental natural frequencies of the system are correspondingly 17, 13.3, 11.4, and $9.2 \mathrm{~Hz}$. This result is slightly different compared to results of modal analysis (i.e., 16.5, 14.5, 11.8, and $9.5 \mathrm{~Hz}$ ). That is, the resonance frequency decreases as the location of the mass gets farther. Thus, we can readily design the harvester having a specific frequency by changing the location of the mass on the rigid bar. Time history responses for resonant frequency of each case are represented in order to clarify the type of output signal in Figure 4. The output voltages are normalized by acceleration of excitations. All of output signals are clearly measured in the sinusoidal wave.

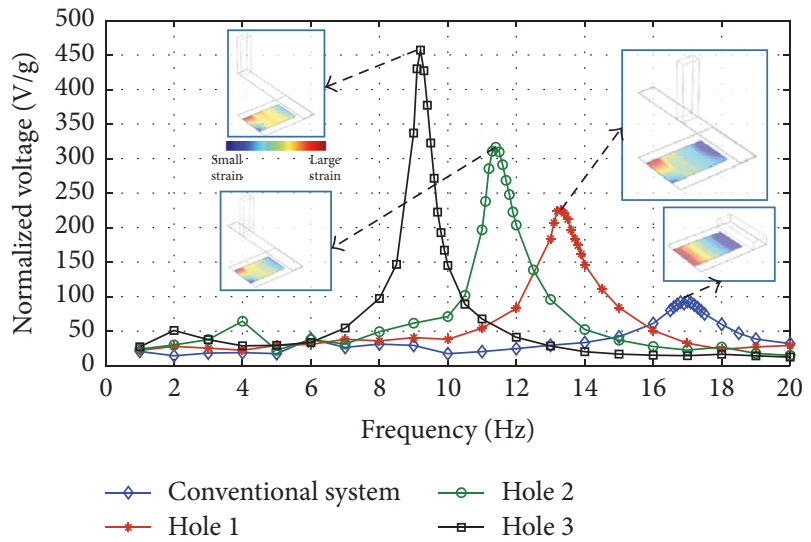

FIGURE 5: Normalized output voltages from experiments: insets are the relative total strains at resonance from FEM simulations.

In the bandwidth, the normalized voltage of the conventional system at $3 \mathrm{~dB}$ below the peak is $65.6 \mathrm{~V} / \mathrm{g}$. The three cases of the proposed system (i.e., Hole 1, Hole 2, and Hole 3) show significant increases in bandwidth of 234.3, 228.6, and $208.6 \%$, respectively, compared to the conventional system. Insets in Figure 5 are the relative total strains at resonance frequency of each case from FEM simulations. The analysis results are carried out considering the position of a proof mass in the static state. Conventional system shows that the 


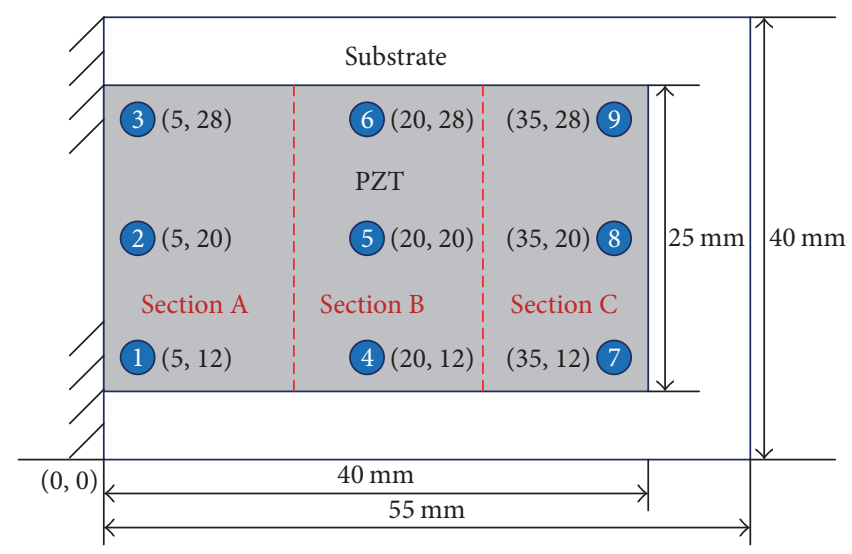

FIGURE 6: The nine virtual nodes in order to obtain stress in FEM simulation.

distribution of strain decreased gradually as it goes away from the fixed end. However, the L shape energy harvester can confirm that strain is evenly distributed. In proposed system, the strain is canceled from fixed end but on the overall average is larger than strain of conventional system. As a result, the proposed energy harvester can produce the high output voltage. Absolute values of each case are compared in the next section.

\section{Numerical Simulation}

In this section, the numerical simulations by using COMSOL Multiphysics are conducted to clearly show the behavior of proposed energy harvester. The COMSOL Multiphysics can help to solve partial differential equation for coupled systems which are electrical, mechanical, fluid, and so on. In Figure 5, the four insets represent the normalized strain distributions obtained from FE analysis in the four different systems. However, the levels of strain in each inset are not normalized. Because an $\mathrm{L}$ shape energy harvester has relatively many large strains, it is difficult to represent the strain of cantilevered type energy harvester. It is observed that the results from the proposed systems are quite different from that of the conventional system; on the other hand, the three different cases in the proposed systems look similar to one another. In general, the cantilever beam type is commonly used in piezoelectric energy harvesting because of the simplicity and the high averaging strain. However, the conventional system has low efficiency because the strain distribution is not consistent. The proposed energy harvester can solve these problems as FE analysis results.

In order to profoundly discuss the experimental results, additional numerical simulations are carried out. In the simulations, the nine virtual nodes on the surface of the piezoelectric sheet are considered as shown in Figure 6. In the figure, all the nodes have the identification numbers from 1 to 9 with $x$ and $y$ coordinates in parentheses. In addition, the piezoelectric sheet is divided into three sections such as Sections A, B, and C according to the distance from the fixed end of the cantilevered beam.
In the simulations, the von Mises stresses at the nodes are calculated. If the stress distribution is assumed to be unidirectional, the output voltage from the piezoelectric plate is known to be generally proportional to the stress of the plate in the condition. Figure 6 shows the von Mises stresses at the nodes in the four different cases.

As shown in Figure 7(a), the conventional system has the constant stresses in the same section (e.g., Nodes 1, 2, and 3 in Section A) and the area close to the fixed end (i.e., Section A) has larger stresses than the area away from the fixed end (i.e., Section C). This is because there is a simple bending mode alone in the conventional system. On the other hand, in the other three cases (see Figures 7(b)-7(d)), a drastic change of stresses among the three nodes is observed in Section A and all the three values are larger than the conventional case. In Sections B and C, the three values are not quite different from one another even though all the values are much larger than the conventional case. Definitely, these larger stress values in all sections affect the high output voltage in the proposed system.

\section{Conclusions}

In this paper, an L shape energy harvester deploying a perpendicular rigid bar with a proof mass is proposed and investigated. Due to the attached rigid bar having a proof mass, the device undergoes the coupled bending and torsional motions, resulting in the frequency shift and the enhanced performance. To verify the effectiveness of the proposed energy harvester, it is simplified to a 2DOF model and its dynamics are described, and the experimental test by using a vertical shaker and the numerical simulation by FE analysis software are conducted. It is demonstrated that the fundamental frequency of the system decreases and the output voltage from the system gets higher as an increase in the arm length. Moreover, the reason of the enhanced performance of the proposed system is discussed by examining the FE stress analysis results. It is concluded that the proposed energy harvester has the frequency tuning capability and the enhanced performance compared with the conventional one. 


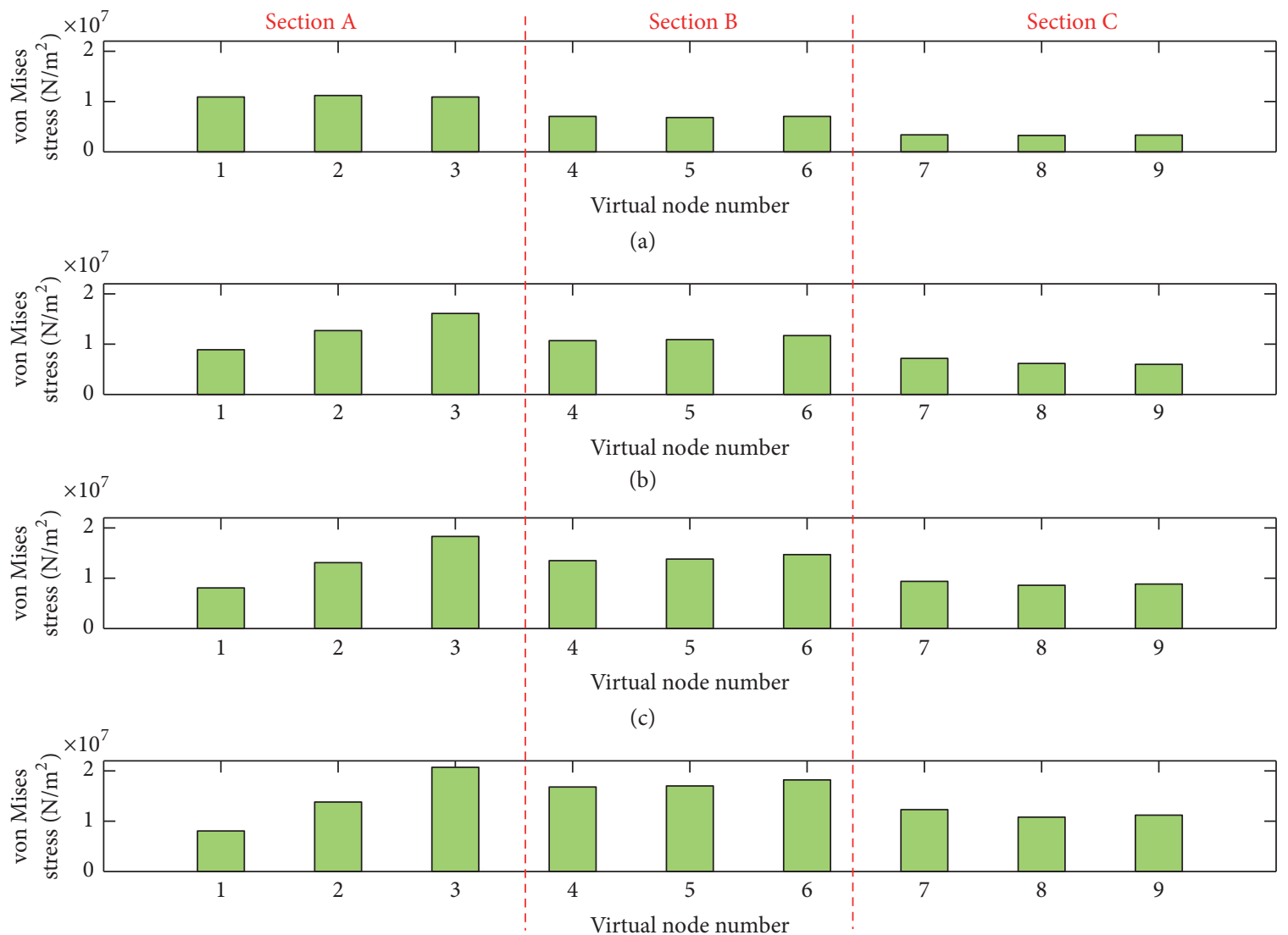

(d)

Figure 7: von Mises stresses from numerical simulation: (a) conventional system; (b) Hole 1; (c) Hole 2; (d) Hole 3.

\section{Conflicts of Interest}

The authors declare that they have no conflicts of interest.

\section{Acknowledgments}

This research was supported by Smart Civil Infrastructure Research Program (Grant 17SCIP-C116873-02) and Technology Advancement Research Program funded by Ministry of Land, Infrastructure and Transport (MOLIT) of Korean government (Grant 17CTAP-C129736-01).

\section{References}

[1] A. Nechibvute, A. Chawanda, and P. Luhanga, "Piezoelectric energy harvesting devices: an alternative energy source for wireless sensors," Smart Materials Research, vol. 2012, Article ID 853481, 13 pages, 2012.

[2] A. Erturk and D. J. Inman, Piezoelectric Energy Harvesting, John Wiley \& Sons, Hoboken, NJ, USA, 2011.

[3] Z. Saadatnia and E. Esmailzadeh, "Nonlinear harmonic vibration analysis of fluid-conveying piezoelectric-layered nanotubes," Composites Part B: Engineering, vol. 123, pp. 193-209, 2017.

[4] E. Asadi, H. Askari, M. Behrad Khamesee, and A. Khajepour, "High frequency nano electromagnetic self-powered sensor:
Concept, modelling and analysis," Measurement, vol. 107, pp. 3140, 2017.

[5] S. P. Beeby, R. N. Torah, M. J. Tudor et al., "A micro electromagnetic generator for vibration energy harvesting," Journal of Micromechanics and Microengineering, vol. 17, no. 7, pp. 12571265, 2007.

[6] H.-J. Jung, I.-H. Kim, and S.-J. Jang, "An energy harvesting system using the wind-induced vibration of a stay cable for powering a wireless sensor node," Smart Materials and Structures, vol. 20, no. 7, Article ID 075001, 2011.

[7] G. Zhu, Z.-H. Lin, Q. Jing et al., "Toward large-scale energy harvesting by a nanoparticle-enhanced triboelectric nanogenerator," Nano Letters, vol. 13, no. 2, pp. 847-853, 2013.

[8] Z. L. Wang, "Triboelectric nanogenerators as new energy technology for self-powered systems and as active mechanical and chemical sensors," ACS Nano, vol. 7, no. 11, pp. 9533-9557, 2013.

[9] A. Ahmed, Z. Saadatnia, I. Hassan et al., "Self-Powered Wireless Sensor Node Enabled by a Duck-Shaped Triboelectric Nanogenerator for Harvesting Water Wave Energy," Advanced Energy Materials, vol. 7, no. 7, 2017.

[10] H. Askari, E. Asadi, Z. Saadatnia, A. Khajepour, M. B. Khamesee, and J. Zu, "A hybridized electromagnetic-triboelectric selfpowered sensor for traffic monitoring: concept, modelling, and optimization," Nano Energy, vol. 32, pp. 105-116, 2017. 
[11] N. M. White, P. Glynne-Jones, and S. P. Beeby, "A novel thick-film piezoelectric micro-generator," Smart Materials and Structures, vol. 10, no. 4, pp. 850-852, 2001.

[12] H.-B. Fang, J.-Q. Liu, Z.-Y. Xu et al., "Fabrication and performance of MEMS-based piezoelectric power generator for vibration energy harvesting," Microelectronics Journal, vol. 37, no. 11, pp. 1280-1284, 2006.

[13] S. Priya and D. J. Inman, Energy Harvesting Technologies, Springer, New York, NY, USA, 2009.

[14] I.-H. Kim, H.-J. Jung, B. M. Lee, and S.-J. Jang, "Broadband energy-harvesting using a two degree-of-freedom vibrating body," Applied Physics Letters, vol. 98, no. 21, Article ID 214102, 2011.

[15] I.-H. Kim, S. Jin, S.-J. Jang, and H.-J. Jung, "A performanceenhanced energy harvester for low frequency vibration utilizing a corrugated cantilevered beam," Smart Materials and Structures, vol. 23, no. 3, Article ID 037002, 2014.

[16] M.-H. Seo, D.-H. Choi, I.-H. Kim, H.-J. Jung, and J.-B. Yoon, "Erratum: Multi-resonant energy harvester exploiting highmode resonances frequency down-shifted by a flexible body beam (Applied Physics Letters (2012) 101 (123903))," Applied Physics Letters, vol. 101, no. 17, Article ID 179905, 2012.

[17] H. Wu, L. Tang, Y. Yang, and C. K. Soh, "A Compact 2 Degreeof-Freedom Energy Harvester with Cut-Out Cantilever Beam," Japanese Journal of Applied Physics, vol. 51, no. 4R, Article ID 040211, 2012.

[18] M. A. Karami and D. J. Inman, "Electromechanical modeling of the low-frequency zigzag micro-energy harvester," Journal of Intelligent Material Systems and Structures, vol. 22, no. 3, pp. 271282, 2011.

[19] S. Li, J. Yuan, and H. Lipson, "Ambient wind energy harvesting using cross-flow fluttering," Journal of Applied Physics, vol. 109, no. 2, Article ID 026104, 2011.

[20] A. Abdelkefi, F. Najar, A. H. Nayfeh, and S. B. Ayed, "An energy harvester using piezoelectric cantilever beams undergoing coupled bending-torsion vibrations," Smart Materials and Structures, vol. 20, no. 11, Article ID 115007, 2011.

[21] A. Abdelkefi, A. H. Nayfeh, M. R. Hajj, and F. Najar, "Energy harvesting from a multifrequency response of a tuned bendingtorsion system," Smart Materials and Structures, vol. 21, no. 7, Article ID 075029, 2012.

[22] H. D. Akaydin, N. Elvin, and Y. Andreopoulos, "Energy harvesting from highly unsteady fluid flows using piezoelectric materials," Journal of Intelligent Material Systems and Structures, vol. 21, no. 13, pp. 1263-1278, 2010.

[23] H. D. Akaydin, N. Elvin, and Y. Andreopoulos, "The performance of a self-excited fluidic energy harvester," Smart Materials and Structures, vol. 21, no. 2, Article ID 025007, 2012. 


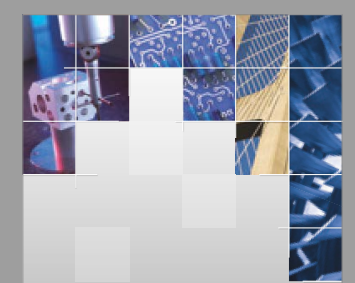

\section{Enfincering}
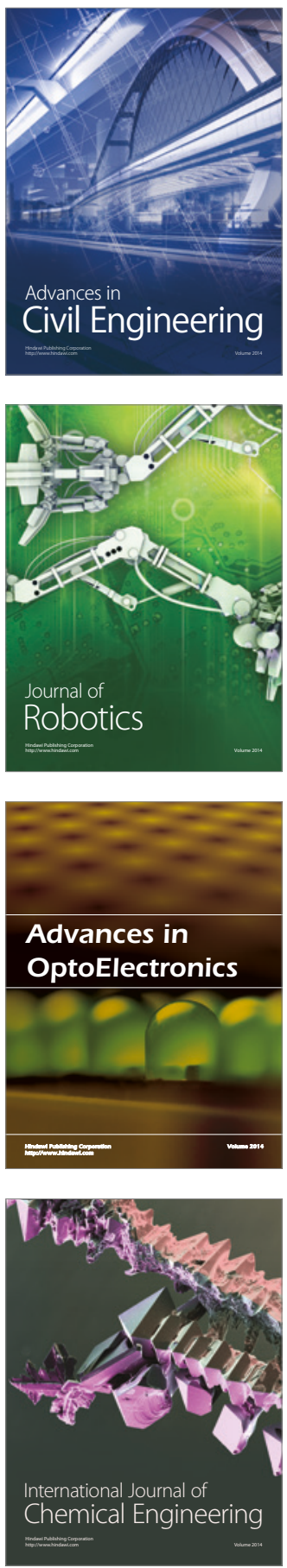

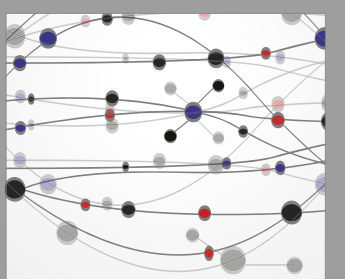

The Scientific World Journal

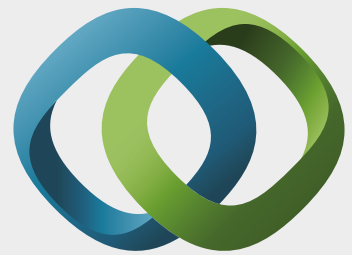

\section{Hindawi}

Submit your manuscripts at

https://www.hindawi.com
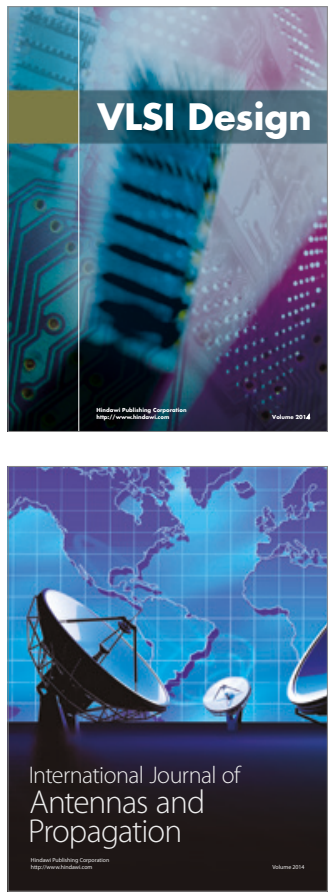

\section{Rotating}

Machinery
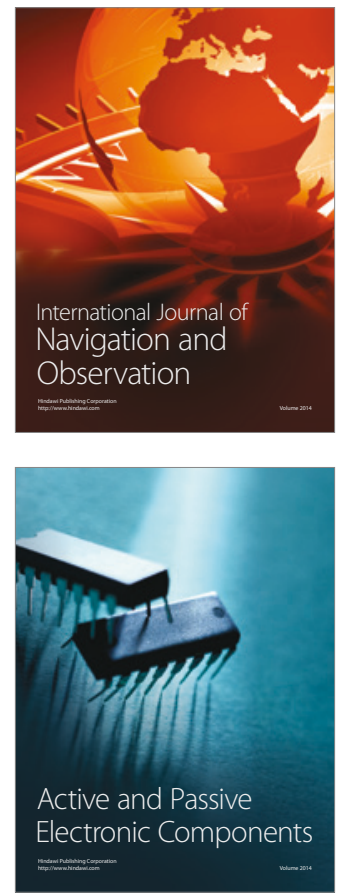
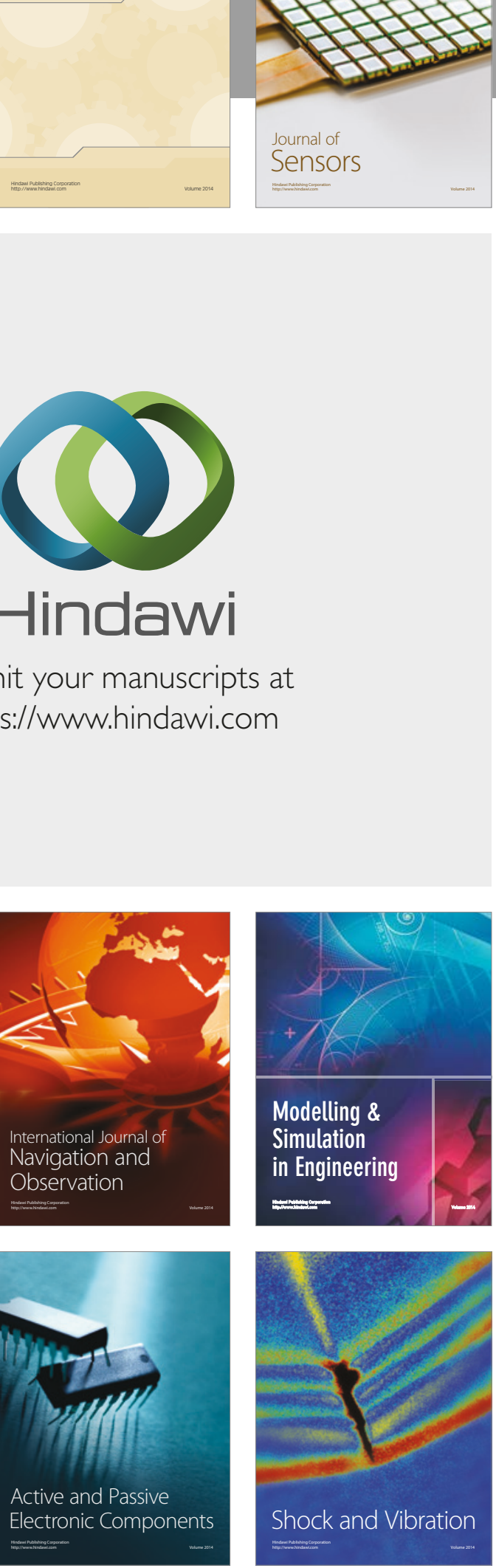
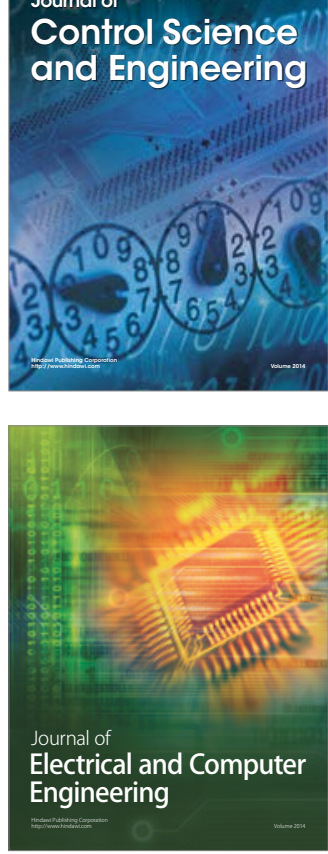

Distributed

Journal of

Control Science

and Engineering
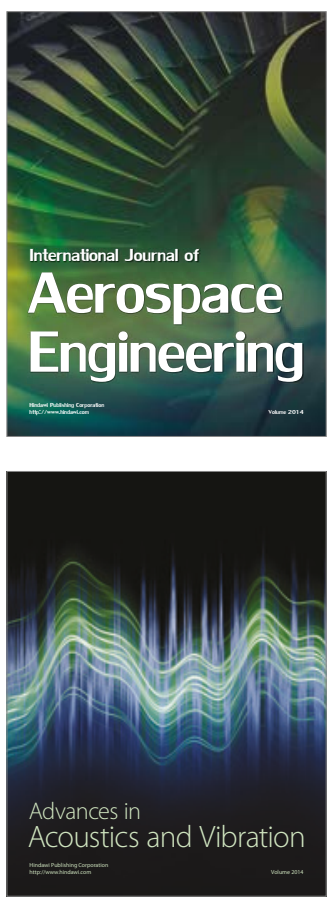

Sensor Networks 